\title{
Anabases
}

ANABASES Traditions et réceptions de l'Antiquité

$1 \mid 2005$

Varia

\section{Les Anciens connaissaient-ils l'Amérique? Une question controversée aux XVIe et XVIIe siècles}

\section{Germaine Aujac}

\section{(2) OpenEdition}

1 Journals

Édition électronique

URL : http://journals.openedition.org/anabases/1446

DOI : 10.4000/anabases. 1446

ISSN : 2256-9421

Éditeur

E.R.A.S.M.E.

\section{Édition imprimée}

Date de publication : 1 mars 2005

Pagination : 163-191

ISSN : 1774-4296

\section{Référence électronique}

Germaine Aujac, "Les Anciens connaissaient-ils I'Amérique? Une question controversée aux XVle et XVIle siècles », Anabases [En ligne], 1 | 2005, mis en ligne le 01 septembre 2011, consulté le 20 octobre 2019. URL : http://journals.openedition.org/anabases/1446 ; DOI : 10.4000/anabases.1446 
Anabases 1 (2005), p. 163-191

\section{Les Anciens connaissaient-ils l'Amérique? Une question controversée aux XVIe et XVII' siècles}

GERMAINE AUJAC

LE PLANISPHÈRE ${ }^{1}$ imprimé à Padoue en 1700, qui avait été mis en grec par Chrysanthos Notaras ${ }^{2}$, "prêtre et archimandrite du Très Saint Siège patriarcal et apostolique de Jérusalem", portait, dans l'hémisphère représentant le Nouveau Monde, la mention explicite, "Atlantis, l'île de Platon». Cette carte du monde ancien et nouveau illustrait l'Introduction à la Géographie et à la Sphérique (en grec) qui fut publiée à Paris en 1716; l'un des chapitres de ce traité (section IV, chapitre 19) posait la question: «Si les Anciens ont connu l'Amérique». Répondant par l'affirmative, Chrysanthos Notaras évoquait entre autres les arguments proposés par le géographe allemand Philippe Cluvier (1580-1623), auteur d'une Introductio in universam geographiam tam veterem quam novam, parue à Leyde en 1624 et maintes fois rééditée. Il y ajoutait ceux proposés par le Hollandais Gérard Jean Vossius (1577-1649), qui, dans son De quatuor artibus popularibus, publié à Amsterdam en 1650, se montrait pourtant sceptique sur la validité des hypothèses formulées par ceux qui tenaient à démontrer que les Anciens aient pu connaître l'Amérique.

Dans les siècles qui suivirent la découverte du Nouveau Monde en effet, nombreux furent ceux qui, géographes, philologues, érudits, hommes de lettres, prirent parti dans ces débats en s'appuyant sur certains passages des textes anciens, grecs ou latins, voire bibliques, auxquels on attribuait souvent une valeur prophétique ${ }^{3}$. Après avoir rappelé quelques-uns des auteurs qui sont intervenus dans cette controverse, nous examinerons les œuvres ou plutôt les fragments d'œuvres le

1 Sur ce planisphère, cf. G. AUJAC, "Cartes géographiques en grec moderne imprimées à Padoue en 1700", Geographia Antiqua 6 (1997), p. 165-181.

2 Chrysanthos NOTARAS (c. 1665-1733), neveu du patriarche de Jérusalem Dosithée auquel il succéda en 1707, fit des études à Constantinople, les compléta de 1697 à 1700 à Padoue, puis, sur le chemin du retour, passa par Paris où il fut accueilli par Cassini à l'Observatoire.

3 Cf. O. BOURA, Les Atlantides. Généalogie d'un mythe, Paris, Arléa, 2003. 
plus souvent cités à l'appui de leurs thèses; nous nous demanderons alors pourquoi d'autres textes, plus explicites, n'ont pas été versés au dossier.

\section{Quelques acteurs de la controverse}

Est-il besoin de rappeler que Christophe Colomb, découvrant de fait un monde encore inconnu, pensait avoir tout simplement atteint les Indes, dont les cartes de Ptolémée avaient répandu la connaissance? D’où le nom d'Indes Occidentales donné d'abord à l'Amérique, et qui persista assez longtemps, renforcé par la similitude que l'on constatait entre le climat et la faune des deux Indes.

L'un des premiers à décrire le Nouveau Monde fut le Jésuite Gonzalo Fernandez de Oviedo (1478-1557) qui avait gagné les Indes en 1514 et y était revenu à plusieurs reprises, principalement en Colombie et à Saint-Domingue. Après un Sumario de la natural historia de las Indias, publié en 1526, à Tolède (traduit en italien par Batisto Ramusio en 1534), il produisit une Historia general y natural de las Indias, islas y Tierra Firma del mar Oceano en vingt livres, qui parut en 1535, à Séville ${ }^{4}$ : il y prenait Pline l'Ancien pour modèle et décrivait avec minutie la flore et la faune qu'il avait pu observer en Amérique centrale, ainsi que les mours des habitants. Contre Pline pourtant, qui croyait la zone torride inhabitable, Oviedo soutenait qu'elle était habitée, "comme nous en avons fait l'expérience dans la Terre Ferme ${ }^{5}$ des Indes» (Historia General II, 1). Persuadé que les Anciens ont su où étaient ces Indes, il voyait dans l'île au delà de Gibraltar, découverte par des Carthaginois et citée par Aristote - à qui il attribuait le De Mundo -, «l'une de celles qui sont en nos Indes de par deça, comme Hispaniola, Cuba, ou peut-être une partie de la Terre Ferme». Mais soucieux de légitimer la domination espagnole sur le Nouveau Monde, il soutenait que «ces Indes sont les fameuses îles Hespérides, ainsi appelées du douzième roi d'Espagne nommé Hespérus», des Hespérides que Solin ${ }^{6}$, dans ses Merveilles du Monde (chap. 68), plaçait à quarante jours de navigation des Gorgones (sc. Cap Vert).

Francisco Lopez de Gomara (né en 1510 à Séville), publiant en 1552 à Saragosse ${ }^{7}$ une Historia de las Indias, s'inscrivait en faux contre l'identification du Nouveau Monde aux Hespérides. Après avoir rappelé ce que Platon dit de l'Atlantide

4 Cf. G.F. DE OVIEDO, Historia general y natural de las Indias, éd. J. PEREZ DE TUDELA BUESO, Biblioteca de Autores Espanoles, 117-121, Madrid, Atlas, 1959.

5 La Terre Ferme était, par opposition aux îles, le littoral de la mer des Caraïbes, vers la Colombie ou le Vénézuela.

6 G.J. Solin (fl. 200 ap. J.-C.), auteur d'un résumé géographique du monde connu dont les principaux éléments sont empruntés à Pline l'Ancien et Pomponius Mela

7 Cf. F.L. DE GOMARA, Primera parte de la Historia general de las Indias, p.155-294 in Biblioteca de autores Espanoles, Historiadores primitivos de Indias, t. XXII, éd. Enrique de Vedia, Madrid, Atlas, 1946. Ce livre fut traduit en italien et publié à Rome en 1556, à Venise, en 1560 et 1565; traduit en français par Martin Fumée (à qui est empruntée la traduction), il fut publié à Paris en 1578, 1584, 1587, 1597 et 1605 . 
dans le Timée et le Critias, il ajoutait (p. 292): «Aujourd'hui, il ne faut plus disputer ni douter de cette île Atlantide, puisque la découverte et la conquête de nos Indes éclaircissent entièrement ce que Platon a écrit [...] Ainsi nous pouvons dire que nos Indes sont l'île et terre ferme de Platon, et non les Hespérides, ni Ophir ni Tharsis 8 comme certains modernes l'ont prétendu». Au reste, le poète Sénèque ${ }^{9}$ n'a-t-il pas été bon prophète «en sa tragédie de Médée, touchant ce Nouveau Monde que nous appelons les Indes? Car il me semble que cette découverte confirme ses propos point par point, et que nos Espagnols et Christophe Colomb ont réalisé sa prophétie». Bien des raisons donc pour croire que les Anciens ont connu ou pressenti l'existence du Nouveau Monde. Gomara, qui fut chapelain de Fernand Cortez, le conquérant du Mexique, consacra la seconde partie de la Cronica general de las Indias au récit de cette conquête.

Un peu plus tard, José de Acosta (1539-1600) qui avait passé une quinzaine d'années au Pérou ${ }^{10}$, principalement dans un collège de jésuites installé sur les bords du lac Titicaca, et puis trois ans à Mexico, composa en latin, du temps qu'il était au Pérou, les deux premiers livres de son Historia Natural y Moral de las Indias qui en contient sept ${ }^{11}$. Il y évoquait les opinions des Anciens, dont certains refusaient de croire à l'existence d'un Monde inconnu, peuplé d'habitants, alors que les philosophes en général n'excluaient pas cette possibilité. Y avait-il des antipodes ${ }^{12}$ ? la zone torride ${ }^{13}$ était-elle inhabitable? pouvait-on traverser l'Océan? Autant de questions qui avaient reçu dans l'Antiquité des réponses diverses ${ }^{14}$. José de Acosta les discute, textes anciens à l'appui.

8 Ophir et Tharsis sont des lieux fabuleusement riches, évoqués dans la Bible, où le roi Salomon envoyait sa flotte pour en rapporter or, argent et autres denrées précieuses.(2Chron. ou 2 Paralipomènes IX, 10 et 21).

9 Les vers de la Médée de Sénèque seront présentés dans la seconde partie de l'article.

10 Le Pérou avait été découvert en 1528; en 1535, Pizarro fondait Lima. José de Acosta avait appris suffisamment de Aymara et de Quechua pour composer un catéchisme trilingue (1583).

11 Les livres suivants furent rédigés en Espagne, en espagnol. Les livres III et IV portent sur la géographie physique et les ressources du pays, les livres V à VII traitent de géographie humaine. Cf. Obras del P. Jose de Acosta dans Biblioteca de Autores Españoles, Madrid, Atlas, 1954, et J. DE ACOSTA, Histoire naturelle et morale des Indes Occidentales trad. de J. Remy-Zéphir, Paris, Payot, 1979.

12 Cf. Historia I, 7-8 ; Lactance, l'apologiste chrétien mort en 323, niait leur existence. Saint Augustin (354-430), dans la Cité de Dieu (XVI, 9), n’y voyait qu'une simple hypothèse logique, sans aucun lien avec l'expérience.

13 Cf. Historia I, 9-10. Les Anciens répartissaient le globe terrestre en cinq zones, deux zones glaciales, autour des pôles, inhabitables par suite du froid, une zone torride entre les tropiques, inhabitable par suite de la chaleur, et deux zones tempérées, les seules considérées comme habitables. Pour les discussions passionnées dans l'Antiquité à propos de l'extension de ces zones, cf. Strabon, Géographie II, 2, 2 - 3, 2.

14 Les textes anciens invoqués, qui sont tous à peu près les mêmes chez les divers auteurs, seront examinés ensemble dans la seconde partie. 
Mais, si tous les hommes descendent d'Adam et Ėve, comme le veut l'Écriture, comment le Nouveau Monde a-t-il pu être peuplé? La traversée par mer étant pratiquement impossible avant la découverte de la pierre d'aimant (Hist. I, 16-17), l'on peut imaginer que des hommes furent jetés par la tempête et contre leur volonté sur les rivages inconnus (Hist. I, 19), mais il est plus probable que les premiers habitants des Indes occidentales y sont arrivés par la terre (Hist. I, 20): «Je conjecture que le Nouveau Monde, que nous appelons Indes, n'est pas complètement distinct de l'Ancien ni séparé de lui... Je tiens que l'une et l'autre terre, en quelque endroit, se joignent et se continuent, ou pour le moins s'avoisinent et s'approchent beaucoup.» Quant au récit de Platon sur l'Atlantide, Acosta le juge incroyable ${ }^{15}$, comme il récuse les interprétations de Proclus, Porphyre ou Origène, qui insistent sur la véracité de l'histoire racontée à Solon par les prêtres égyptiens: ce n'est en tout cas pas par cette île, disparue, que les Indiens ont pu arriver dans le Nouveau Monde (Hist. I, 22). Si l'Atlantide a existé et puis a été engloutie, elle ne peut être l'Amérique.

Au livre II, José de Acosta dresse un tableau idyllique de cette zone dite, bien à tort, torride par les Anciens: elle est en fait de climat tempéré et beaucoup moins sèche qu'ils ne l'ont représentée. D'où il conclut: "Ce que les poètes chantent des Champs-Élysées et de la fameuse Tempé ${ }^{16}$, et ce que Platon nous dit ou laisse croire au sujet de sa fameuse Atlantide, les hommes le trouveraient certainement dans ces terres-là, si d'un cœur généreux ils préféraient être maîtres, plutôt qu'esclaves, de leur argent et de leur cupidité» (Hist. II, 14).

L' Histoire Naturelle et Morale des Indes, l'un des premiers ouvrages scientifiques sur le Nouveau Monde, eut grand succès. Publiée en espagnol à Séville en 1589, elle fut traduite en italien (1596), français (1598), allemand (1598), anglais (1604), hollandais (1698) et latin (1590). Les géographes postérieurs lui doivent une bonne partie de leur savoir.

C'est dans ses Relazioni Universali, publiées à Vicenza en 1595 (en trois parties), puis à Brescia en 1599 (en quatre parties), et surtout à Venise en 1599, 1602, 1607, etc., que l'Italien Giovanni Botero (1540-1617), qui lui n'avait jamais quitté l'Europe ${ }^{17}$, entrait à son tour dans l'arène. La première partie des Relazioni offrait les descriptions de l'Europe (l. I), de l'Asie (l. II), de l'Afrique (l. III), chacune étant accompagnée de la carte correspondante. Le livre IV de cette première partie s'ouvrait sur la fameuse question controversée: «Si les Anciens avaient connaissance du Nouveau Monde». L'argumentation utilisait les thèmes habituels, considérant que le

5 Il ajoute: «Quant à moi, je n’ai pas pour Platon une telle révérence» ( Hist. I, 22).

16 C'est une vallée de la Grèce, entre l'Olympe et l'Ossa, dont Virgile a chanté la beauté.

17 G. Botero était surtout connu de son temps pour ses opinions politiques et ses études économiques. Son célèbre De la raison d'État publié à Venise en 1589 et 1619, à Turin en 1596, fut traduit en plusieurs langues dont en français en 1606 par Pierre de Deimier. Un autre traité, Des causes de la grandeur des cités (Rome, 1598), semble avoir eu moins de succès. 
Monde Nouveau était situé dans la zone torride ${ }^{18}$, bien au delà de Gibraltar. Et cette zone torride est habitée, contrairement à l'opinion d'Aristote et de beaucoup d'Anciens. D'ailleurs Pline qui raconte les périples d'Hannon le Carthaginois et d'Eudoxe de Cyzique autour de l'Afrique, et qui décrit l'̂̂le de Taprobane ${ }^{19}$, savait bien, précise Botero, qu'une grande partie de la zone torride est habitable. Comme d'Acosta, G. Botero souligne que les Anciens n'avaient aucun moyen de traverser l'Océan, vu qu'ils ignoraient les pouvoirs de l'aiguille aimantée, révélée seulement aux Européens vers 1300. Mais il contestait les dires de son prédécesseur, nommément cité, sur le climat tempéré du Nouveau Monde, et sur ses ressources, supérieures à celles de l'Ancien Monde. En revanche, il développait les mêmes hypothèses que lui sur les origines du peuplement de l'Amérique, concluant avec lui que les habitants de ce continent longtemps inconnu étaient venus d'Europe ou d'Asie par des voies terrestres, au nord ou au sud ${ }^{20}$.

Oviedo, Gomara, de Acosta et Botero, les Espagnols et l'Italien, étaient unis par une même foi et par une formation similaire à l'école des Jésuites. Leur œuvre, riche en renseignements de toutes sortes, fait une large place aux considérations religieuses. Tout autre est le projet de l'allemand Philippe Cluvier (1580-1623), dont l'Introductio in universam geographiam tam veterem quam novam est devenue très vite l'ouvrage de référence pour quiconque s'occupait de géographie.

Né à Danzig, et après des voyages en Allemagne et en Pologne, Cluvier commença des études de droit à Leyde, mais se passionna bientôt pour l'histoire et la géographie, enseignées par Joseph Scaliger, philologue protestant (1540-1609). Il participa à des campagnes en Bohème et Hongrie, fut fait prisonnier, voyagea ensuite en Angleterre, Écosse, France, Italie pour se fixer définitivement en Hollande où,

18 Les trois historiens du Nouveau Monde présentés ici étaient des Espagnols, familiers pour l'essentiel des territoires découverts et occupés par l'Espagne en Amérique latine, Pérou, Colombie, Mexique, Panama. Botero leur emprunte sa connaissance du Nouveau Monde.

19 L'île de Taprobane est l'actuelle Sri Lanka (Ceylan), que les géographes grecs plaçaient vers $12^{\circ} \mathrm{N}$ (cf. Strabon, Géogr. II, 5, 35). Elle se trouve en fait entre $7^{\circ}$ et $10^{\circ} \mathrm{N}$. Ovide, dans les Pontiques I, 5, soulignait son excentricité: «Quel intérêt pour toi d'être loué par la brûlante Syène (sc. Assouan), ou par les lieux où l'Océan Indien baigne Taprobane?» (v. 79-80). D’après Pline (Hist. Nat. VI, 81), Taprobane avait longtemps été considérée comme un autre monde et qualifiée de terre des Antichthones, ce qui la situait dans l'hémisphère sud. D'Acosta qui en parle aussi l'identifiait à Sumatra. Sur cette identification, fréquente à la Renaissance, cf. M.T. GAMBLIN, "L'île Taprobane: problèmes de cartographie dans l'Océan Indien”, in Géographie du monde au Moyen Âge et à la Renaissance, éd. M. PELLETIER, Paris, éditions du C.T.H.S., 1989, p. 191-200.

20 Les deux derniers livres de cette première partie sont consacrés à la description du Nouveau Monde. La deuxième partie traite des principaux princes d'Europe, d'Asie, d'Afrique, avec un livre spécial pour les pouvoirs du Pape, du Roi Catholique et du Grand Turc. La troisième partie analyse les croyances et les religions des divers peuples connus. La quatrième partie porte sur le Nouveau Monde, sa connaissance de Dieu, son ignorance de l'Évangile et les efforts pour y remédier 
après 1616, l'Académie de Leyde lui accorda une pension régulière qui lui permit de se consacrer à la rédaction et à la publication de ses ouvres: Germania Antiqua en 1616, Siciliae antiquae libri duo, Sardinia et Corsica antiqua en 1619, Italia antiqua, publiée après sa mort en 1624, et finalement sa grande Introductio, en six livres ${ }^{21}$, publiée à Leyde en 1624.

Philippe Cluvier, comme on a pu s'en apercevoir par le titre de ses publications antérieures, est surtout intéressé par l'ancien monde. Le livre I de l'Introductio résume l'enseignement des Anciens en matière de géographie mathématique; traitant de la navigation sur l'Océan, il fait référence aux périples d'Hannon et d'Eudoxe évoqués par Pline (Hist. nat., II, 169). Les livres II, III et IV contiennent une description détaillée de l'Europe, «la plus fameuse et célèbre partie de tout le monde, tant pour le fait des armes que pour l'honneur de la littérature» (II, 1). Le livre V est consacré à l'Asie, le livre VI à l'Afrique et à l'Amérique. Ainsi, des six livres de cette somme géographique si réputée, seule la dernière partie du livre VI traite de l'Amérique.

Au chapitre 11, intitulé "Description générale de l'Amérique», Cluvier déclare: «L'on pourrait tirer quelques preuves des écrits de Platon et de Diodore qu'elle a été anciennement reconnue par les peuples de l'Europe». Il cite le Timée avec le récit sur l'Atlantide, le livre $\mathrm{V}$ de Diodore de Sicile où est évoquée une grande île au-delà des Colonnes d'Hercule qu'auraient atteinte, par mégarde, des Phéniciens emportés par la tempête: "Or cette île, si l'on considère sa situation et sa grandeur, ne peut être autre que celle que l'on nomme vulgairement Amérique." Mais Cluvier continue: «Davantage l'auteur du petit livre $D u$ Monde que l'on attribue à Aristote ou à Théophraste, duquel la version latine a été faite par Apulée, dit qu'il y a d'autres grandes îles outre l'Europe, l'Asie et l'Afrique, et ainsi sans doute ces Anciens ont entendu parler de l'Amérique."

Avant de clore ce chapitre, Cluvier rappelle que «cette terre est nommée par quelques-uns l'Inde Occidentale, d'autant qu'elle fut découverte au même temps que l'Inde orientale, et aussi que les peuples de l'une et de l'autre vivent de même façon; car ils vont les uns comme les autres tout nus.» La grande renommée de Cluvier géographe, qui, lui, ne doutait pas que les Anciens aient pu avoir connaissance d'un continent mystérieux qui aurait été l'Amérique, fit que les textes invoqués en faveur de cette thèse, le Timée, la Bibliothèque de Diodore, le De Mundo, ont été très largement repris et commentés par ses successeurs. Les Tabulae geographicae publiées en 1699 par le Séminaire de Padoue ${ }^{22}$, dont la première partie devait servir «à la géographie en

21 Cette somme géographique eut des éditions nombreuses pendant tout le XVII e siècle, trois à Leyde $(1624,1627,1641)$, dix à Amsterdam $(1629,1651,1659,1661,1663$, $1677,1682,1683,1683,1697)$, quatre à Paris $(1630,1631,1635,1635)$, où fut publiée aussi, en 1642 et 1648, une traduction française de ce traité. Des grands noms participèrent de près ou de loin à ces diverses éditions: P. Bertius, Daniel Heinsius, Jean Bunon, Reiskius.

22 Cf. AUJAC, "Cartes géographiques", p. 169. 
général, mais de préférence à l'illustration de l'Introduction de Cluvier», s'ouvraient par une carte générale en deux hémisphères, dressée par N. Sanson et datée de 1694: l'hémisphère réservé au continent américain, très schématisé, était qualifié de "Atlantis, île de Platon», indication donnée plus tard en grec par Chrysanthos Notaras dans son propre planisphère, imprimé à Padoue en 1700 (cf. planche 5).

Autre acteur important dans la controverse, le théologien protestant Gérard Jean Vossius (1577-1649) qui fit ses études à Leyde, y fut directeur du Collège des États de 1615 à 1618 , puis, après une courte disgrâce ${ }^{23}$, professeur d'histoire à l'Université (1622-1633), avant de terminer sa carrière à Amsterdam.

Cet humaniste hollandais, qui connaissait aussi bien le grec, le latin, l'hébreu, que les mathématiques, la philosophie ou la théologie, est l'auteur de nombreux traités, réunis après sa mort dans un ensemble publié à Amsterdam, chez J. Blaeu, en 1650 et 1660, sous le titre De quatuor artibus popularibus, de philologia et scientiis mathematicis. Les quatre "arts populaires", traités en cinq chapitres, sont la grammaire, la gymnastique, la musique et la peinture. La philologie est détaillée en douze chapitres, dont le onzième est consacré à la géographie. Vossius y déclare (XI, 27): "Il faut connaitre les régions inconnues des Anciens. Autrefois on ne connaissait que trois continents. Il s'y ajoute l'Amérique ou Inde occidentale et la Magellanique ${ }^{24}$ ou terre australe [...] Pour beaucoup d'érudits, les Anciens semblent n'avoir pas totalement ignoré ces deux continents. Ils pensent que se réfere à l'Amérique ce que Platon dit de l'Atlantide, et ce que rapporte Aristote ${ }^{25}$ des expéditions des Phéniciens Gaditains vers une région inconnue et de la navigation des Carthaginois vers une île grande et déserte, mais fertile. De même ils pensent que regarde l'Australe ce que l'on peut lire de l'itinéraire de Iambulos chez Diodore de Sicile au livre II. Même Strabon, au livre II, dit qu'il est vraisemblable qu'il y ait d'autres terres que celles alors connues ${ }^{26}$. Et surtout c'est clair chez Festus Aviénus 27 dans sa description des côtes maritimes.»

Dans la troisième partie, consacrée aux Sciences Mathématiques, le chapitre XLII est réservé à la géographie. Vossius y rappelle (XLII, 10) que «l'autre continent fut ignoré des Anciens mais est connu de nous. Ce qu'on appelle Amérique ou Inde occidentale a été découvert par Christophe Colomb en 1492.» Il fait référence aux

23 Accusé d'être partisan d'Arminius, le théologien protestant dont la doctrine, plus douce que celle de Calvin, était l'objet de maintes controverses, Vossius fut destitué de sa charge, mais rentra en grâce dès 1622 .

24 Cette Magellanique ou terre australe comprenait tout ce qui se trouvait au sud du détroit de Magellan, soit, outre la Terre de Feu, un continent qui se prolongerait jusqu'au pôle sud, selon les cartographes du XVI ${ }^{\mathrm{e}}$ s. (Abraham Ortelius, 1570 et Gérard Mercator, 1587).

25 Vossius fait ici allusion au De Mundo qu'on attribuait souvent, à l'époque, à Aristote.

26 Cf. Diodore de Sicile, Bibl. hist. II, 55-60, et Strabon, Géogr. II, 5, 13.

27 L'édition princeps des Euvres d'Aviénus, procurée par G. Valla et V. Pisani, avait été imprimée à Venise en 1488. Vossius cite les vers 113 à 129 des Ora Maritima. 
vers d'Aviénus, cités dans le De Philologia, évoque l'Atlantide de Platon et profite même de l'occasion pour critiquer la traduction donnée par Marsile Ficin (14331499) de l'un des passages du Timée. Puis il cite Proclus, qui, dans son Commentaire sur le Timée, rappelle l'opinion de Crantor, le premier interprète de Platon, qui tenait pour vraie l'histoire de l'Atlantide. Vossius en appelle aussi aux auteurs qui ont parlé de l'existence d'îles mystérieuses situées au-delà des Colonnes d'Hercule, Aristote dans le De Mirabilibus Auscultationibus, ou Diodore au livre V de la Bibliothèque Historique. Évoquant l'île décrite par Diodore, Vossius précise: «Beaucoup pensent que cette île est l'Amérique», mais lui-même reste sceptique sur l'identification au Nouveau Monde de l'une ou l'autre de ces îles.

Quant à Chrysanthos Notaras (c. 1665-1733), cet érudit grec qui fut patriarche de Jérusalem, il dut rédiger l'essentiel de son Introduction à la géographie et à la sphérique du temps qu'il se trouvait à Padoue, aux entours de 1700, et qu'il y faisait imprimer son planisphère. Dans cet ouvrage de 153 pages, réparties en quatre sections, c'est la sphérique qui se taille la part du lion, occupant les trois premières sections. La section IV comprend d'abord une partie théorique, confection des cartes, évaluation des distances, orientation, etc. (p. 117-139); c'est seulement dans les treize dernières pages (140-153) que sont décrits succinctement les continents, Europe, Asie, Afrique, Amérique; au $19^{\mathrm{e}}$ et dernier chapitre (p. 149-153), Notaras pose enfin la question: «Si les Anciens connaissaient l'Amérique».

Il reprend d'abord les arguments évoqués par Philippe Cluvier : l'île Atlantide, l'île mystérieuse découverte par les Phéniciens chez Diodore de Sicile, les grandes îles évoquées dans le De Mundo, attribué soit à Aristote soit à Théophraste. "Cluvier en conclut que les Anciens connaissaient une autre terre que l'Ancien Monde, et ce pouvait être l'Amérique.»

D'autres auteurs pourtant, d'opinion contraire, soutiennent que l'Amérique fut ignorée des Anciens, et invoquent à l'appui de leur thèse les silences de Ptolémée et de Strabon. Mais Chrysanthos est persuadé qu'ils ont tort et il en donne des preuves. Strabon par exemple a clairement affirmé ses intentions quand il proclame : «Le but du géographe est de décrire le monde habité dans ses parties connues, de négliger les contrées inconnues, de même que ce qui se trouve en dehors de lui.» (Strabon, Géogr. II, 5, 5). Un peu plus loin, comparant la zone tempérée septentrionale, à la surface du globe terrestre, à une tête d'artichaut, le géographe grec précisait la mission dévolue à la géographie : "L'examen détaillé du globe terrestre ou encore de toute la tête d'artichaut pour la zone étudiée relève d'une tout autre science, comme aussi la 
question de savoir si la tête d'artichaut est habitée également dans l'autre quart ${ }^{28}$; en effet, dans l'affirmative, elle n'est certainement pas habitée par des gens de chez nous; il faut alors supposer l'existence d'un autre monde habité, ce qui est plausible. Mais nous, c'est seulement du nôtre que nous avons à parler.» (Strabon, Géogr. II, 5, 13). Voilà donc expliqué le silence volontaire de Strabon sur un continent inconnu dont il considère l'existence plausible, mais qui, de son point de vue, n'a aucun intérêt pour un géographe soucieux d'être utile à ses contemporains et surtout à l'élite des gouvernants.

Après ces objections aux propos de ceux qui refusent de croire que les Anciens aient eu quelque connaissance de ce continent nouveau, Chrysanthos emprunte à Vossius les citations de la Médée de Sénèque et des Ora Maritima de Festus Aviénus. Et il termine en évoquant, à l'appui de sa thèse, ce qui est dit de Tarshish "dans le second livre des Paralipomènes 9, 21»: le navire du roi Salomon y serait allé, puis revenu au bout de trois ans, plein d'or et d'argent ${ }^{29}$. Du coup, «beaucoup ont pensé qu'il s'agissait du Pérou, province d'Amérique du Sud; car du temps de Salomon, l'Amérique était connue.»

Telle n'était pas l'opinion de Mélétios 30 d'Arta (1661-1714), un Grec de Joannina qui, avant d'être archevêque d'Athènes, avait profité d'un séjour forcé de trois ans à Naupacte (1698-1701) pour rédiger en grec une Géographie ancienne et nouvelle qui ne fut publiée qu'après sa mort (Venise, 1728). Après quelques généralités de géographie mathématique (p. 1-41), le livre I décrivait l'Europe (p. 42-441), le livre II l'Asie (p. 442-571), le livre III la Libye (p. 572-608), le livre IV l'Amérique (p.609-620). Dès le premier chapitre du livre IV, Mélétios posait la question: «Cette Amérique fut-elle connue des temps anciens? Cela paraît peu sûr et douteux. Il est plus plausible qu'elle a été totalement ignorée; mais d'autres soutiennent le contraire», en invoquant Platon dans le Timée, Diodore de Sicile au livre V, le De Mundo dont on ne sait si l'auteur est Aristote ou Théophraste. Mais, conclut Mélétios, tous ces arguments sont facilement balayés.

28 Pour Strabon et pour la plupart des géographes grecs antérieurs à Ptolémée, le monde connu n'occupait même pas une moitié de l'hémisphère nord, donc même pas un quart du globe terrestre. D'où la possibilité d'imaginer l'existence d'autres terres inconnues dans les trois autres quarts du globe. L'hypothèse avait été matérialisée sur le globe terrestre fabriqué par Cratès de Pergame (fl. 165 av. J.-C.) à la surface duquel il avait représenté quatre mondes, habités ou non, séparés par des ceintures océaniques infranchissables. De ces quatre mondes imaginaires, un seul, «le nôtre», était connu.

29 José de Acosta avait déjà indiqué «que quelques auteurs ont cru qu'Ofir signifie Pérou dans les Saintes Écritures» (Hist. I, 13); Ofir est ce lieu mystérieux où la flotte de Salomon se rendit et d'où elle rapporta au roi 450 talents d'or (2 Paralipomènes VIII, 18).

30 Mélétios était allé très jeune s'instruire en Vénétie où il fut en contact, par intermédiaire livresque, avec l'essentiel du monde savant. 
Dans un excursus qui se situe à la fin du chapitre consacré aux zones et climats $^{31}$ de la terre, passant en revue les principaux auteurs qui se sont distingués en géographie, Mélétios cite, parmi les modernes, Jean Botero et Philippe Cluvier, ce qui ne nous étonne guère.

Ainsi la question de savoir si l'Amérique fut connue ou non des Anciens, occupait une assez large place dans les discussions des humanistes des XVI ${ }^{\mathrm{e}}$ et XVII ${ }^{\mathrm{e}}$ siècles, encore tout pénétrés de culture classique. Montaigne (1533-1592) lui aussi, dans les Essais parus en 1588, évoquait l'Atlantide de Platon, mais il continuait: "Il n'y a pas grande apparence que cette île soit ce monde nouveau que nous venons de découvrir; car elle touchait quasi l'Espagne, et ce serait un effet incroyable d'inondation de l'en avoir reculée, comme elle est, de plus de douze cents lieues.» (Les Essais, I, 31). Il rappelait un peu plus loin que «l'autre témoignage de l'Antiquité auquel on veut rapporter cette découverte est dans Aristote, au moins si ce petit livre Des merveilles inoü̈es est à lui. Il raconte là que certains Carthaginois, s'étant jetés au travers de la mer Atlantique, hors le détroit de Gibraltar, et navigué longtemps, avaient découvert enfin une grande île fertile, toute revêtue de bois et arrosée de grandes et profondes rivières, fort éloignée de toutes terres fermes [...] Cette narration d'Aristote n'a non plus d'accord avec nos terres neuves.» (I, 31)

\section{Les principaux auteurs invoqués}

Ainsi, ce sont les mêmes passages des mêmes auteurs anciens qui sont cités, à longueur de temps, même si chaque partisan d'une thèse ou de l'autre tentait d'apporter des arguments inédits; mais les textes littéraires, les seuls utilisés par les humanistes (qui se désintéressent des traités scientifiques), ne sont ni très nombreux ni très explicites.

\section{Platon, le Timée et l'Atlantide}

L'Atlantide de Platon est la référence obligée par ceux qui veulent y voir, ou qui refusent d'y voir, le continent nouvellement découvert. Le Timée, l'un des dialogues de Platon ${ }^{32}$ les plus difficiles d'accès, a été étudié et commenté tout au long des siècles. La version latine composée au VIe siècle par Chalcidius, puis celle procurée par Marsile Ficin au XVe siècle, en ont grandement facilité l'accès dans le monde occidental. Le mythe qui l'introduit (20d-25d), relatant l'histoire du continent

31 Les climata sont des bandes parallèles, à la surface du globe terrestre, de largeur variable, qui ont même durée du jour solsticial et sensiblement même hauteur du pôle au dessus de l'horizon. Ptolémée, dans la Syntaxe Mathématique (II, 6), dresse un tableau des climats depuis l'équateur jusqu'au pôle.

32 Cf. Platon, Timée, Critias, éd. et trad. A. RIVAUd, Paris, C.U.F., 1925 (6e tir. 1985) ou Platon, Euvres complètes, trad. L. ROBIN et J. MOREAU, 2 tomes, Paris, Pléiade, 1950. 
disparu, semble d'interprétation relativement aisée; il a pourtant suscité bien des controverses.

«Oyez donc, Socrate, une histoire très singulière mais absolument vraie, à ce que dit une fois Solon, le plus sage des sept sages»(20d): c'est ainsi que Critias annonce le récit qu'il va faire, transmis par Solon qui l'a lui-même reçu de prêtres égyptiens. On y célèbre d'abord les vertus de l'Athènes ancienne, en «un temps, avant la plus grande destruction par les eaux, où la cité qui est aujourd'hui celle des Athéniens était, de toutes, la meilleure dans la guerre et singulièrement la mieux policée à tous les égards» (23c). Parmi les exploits accomplis par cette cité exemplaire, "un surtout l'emporte sur tous les autres en grandeur et en héroïsme. En effet nos écrits rapportent - c'est le prêtre égyptien qui parle - comment votre cité anéantit jadis une puissance insolente qui envahissait à la fois toute l'Europe et toute l'Asie et se jetait sur elles du fond de la mer Atlantique. Car, en ce temps-là, on pouvait traverser cette mer. Elle avait une île, devant ce passage que vous appelez, dites-vous, les colonnes d'Hercule. Cette île était plus grande que la Libye et l'Asie réunies. Et les voyageurs de ce tempslà pouvaient passer de cette île sur les autres îles, et de ces îles, ils pouvaient gagner tout le continent, sur le rivage opposé de cette mer.» (24 e). Les envahisseurs venus de l'Atlantide furent repoussés par les seuls Athéniens qui libérèrent ainsi «tous les autres peuples et nous-mêmes qui habitons à l'intérieur des colonnes d'Hercule. Mais dans le temps qui suivit, il y eut des tremblements de terre effroyables et des cataclysmes. Dans l'espace d'un seul jour et d'une nuit terribles, toute votre armée fut engloutie d'un seul coup sous la terre, et de même l'île Atlantide s'abîma dans la mer et disparut. Voilà pourquoi, aujourd'hui encore, cet Océan de là-bas est difficile et inexplorable, par l'obstacle des fonds vaseux et très bas que l'île, en s'engloutissant, a déposés.» (25c-d). La description détaillée de l'Atlantide, lot de Poséidon, se trouve dans le Critias, dialogue prévu pour être une suite du Timée.

Gomara pense que c'est pour avoir lu le Timée et le Critias que Christophe Colomb est parti à la recherche de la riche Cipango de Marco Polo, et il conclut que les Indes sont l'île ou terre ferme de Platon ${ }^{33}$. José d'Acosta ne cite que le Timée, dans le chapitre 12 du livre I, intitulé: «Du sentiment de Platon à propos de cette Inde Occidentale»; à l'en croire, «si quelqu'un a traité plus particulièrement de cette Inde Occidentale, la gloire en revient à Platon, dans le Timée». Botero ne fait aucune allusion à l'Atlantide, qu'il considère probablement comme une simple fable. Cluvier en revanche résume le passage du Timée évoquant ce continent disparu: il y voit une preuve que l'Amérique a été anciennement reconnue par les peuples de l'Europe

33 GOMARA, Primera parte, p.165, 167, 292. 
(Introductio VI, 11). Quant à Vossius le Hollandais, sans prendre ouvertement parti, il en appelle au Commentaire du Timée par Proclus ${ }^{34}$.

Dans le monde du néo-platonisme en effet, le Timée, comme bien d'autres dialogues de Platon, a été souvent commenté. Certains de ces commentaires sont aujourd'hui perdus, comme celui de Porphyre, mais celui de Proclus a été conservé. On y lisait : «Tout ce discours sur les Atlantins, les uns ont dit que c'est purement et simplement de l'histoire, ainsi Crantor, le premier exégète de Platon ${ }^{35}$ [...] D'autres disent que l'Atlantide est une fable, une fiction qui n'a pas la moindre réalité mais qui comporte une indication sur les réalités éternelles ou soumises au devenir dans le cosmos. Mais ces gens-là n'ont même pas prêté l'oreille à ce que Platon proclame, que "le récit est sans doute très étrange, mais absolument vrai» (20d 8) [...] D’autres ne refusent pas de reconnaître que ces événements se soient passés de cette manière mais ils disent que pour l'instant ils sont amenés comme des images des oppositions préexistantes dans l'univers» (Proclus, Commentaire 76, 1-20, trad. Festugière); parmi ces derniers, Proclus comptait Origène et Porphyre ${ }^{36}$.

Vossius est l'un des rares à utiliser le texte grec du Timée et de ses commentateurs, et à en critiquer au besoin la traduction latine. L'allemand Cluvier s'est probablement contenté de recourir au Timée latin de Chalcidius, publié à Paris en 1520 et à Leyde en 1617, ou plus probablement à la traduction de Marsile Ficin ${ }^{37}$, publiée à Paris en 1536 et 1551 . Il va sans dire que les Grecs, Chrysanthos et Mélétios, lisaient Platon et ses commentateurs dans le texte.

34 Proclus de Lycie (410-485), l'un des personnages influents du néo-platonisme, enseigna à Athènes de 438 à 485. Son Commentaire sur le Timée fut imprimé à Bâle en 1534, et l'édition gréco-latine d'Émile Portus à Hambourg en 1618. Cf. Proclus, Commentaire sur le Timée, trad. A.-J.F ESTUGIÈRE, Paris, Vrin, 1966.

35 Cf. L. TARAN, "Proclus on the old Academy", in Proclus, lecteur et interprète des Anciens, Actes du colloque Paris 1985, publiés par J. PEPIN et H.D. SAFFREY, Paris, éd. du CNRS, 1987, p. 227-276. Pour Crantor, Platon avait attribué le récit sur l'Atlantide à des Égyptiens afin de faire témoigner par les Égyptiens eux-mêmes que l'État idéal présenté dans la République n'était pas emprunté à l'Egypte, comme le prétendaient des adversaires de Platon. D'où ce récit assurant qu'Athènes jadis avait eu une excellente constitution.

36 Origène (185-254), célèbre théologien et exégète des textes bibliques, «disait que le récit était une fiction" (Proclus, Comment. 83, 27). Pour Porphyre (233-305) et son commentaire du Timée, perdu, cf. Porfirio, I frammenti dei commentari al Timeo di Platone, traduzione a cura di A.R. SODANO, Portici (Napoli), Centro Bibliotecario, 1974.

37 Les Euvres complètes de Platon traduites en latin par Marsile Ficin ont fait l'objet de maintes éditions, Venise 1491 et 1581, Florence 1494, Bâle 1532, 1539, 1546, 1561, Lyon 1550 et 1590, Francfort 1602. 


\section{Diodore de Sicile (c. 90-20 av. J.-C.), la Bibliothèque historique}

L'existence d'une grande île au delà des Colonnes d'Hercule est évoquée par Diodore de Sicile ${ }^{38}$ dans sa Bibliothèque Historique, notamment au livre V, 19: «Il y a du côté de la Libye une île de haute mer, de dimensions considérables, située dans l'océan à une distance de la Libye de plusieurs jours de navigation vers l'Ouest. Son sol est fertile, en majeure partie montagneux, mais avec aussi une plaine spacieuse d'une grande beauté. Il y coule des rivières navigables utilisées pour l'irrigation, et l'île contient de nombreux parcs plantés d'arbres d'essences variées et des jardins en quantité, traversés par des courants d'eau douce; on y voit aussi des villas privées, de construction coûteuse, et, à travers ces jardins, des kiosques pour banquets dans des massifs de fleurs; c'est là que les habitants passent leur temps durant l'été, car la terre fournit en abondance tout ce qui contribue aux plaisirs de l'existence et au luxe [...] En général le climat de l'île est si doux qu'il produit en abondance les fruits des arbres et les récoltes saisonnières pendant la majeure partie de l'année, si bien qu'il semblerait que cette île, vu son exceptionnelle félicité, soit la demeure de dieux et non d'hommes.» La description est idyllique ${ }^{39}$; cette île, restée longtemps inconnue, aurait été découverte par des Phéniciens de Gadès, poussés par la tempête. "Les Tyrrhéniens, alors maîtres de la mer, eurent l'intention d'y envoyer une colonie; mais les Carthaginois s'y opposèrent, de peur que beaucoup d'habitants de Carthage n'y émigrent, vu l'excellente qualité de l'île, et aussi pour avoir un lieu où chercher refuge en cas de malheur, si un désastre total faisait périr Carthage. Leur idée était que, maîtres de la mer, ils pourraient tout transporter, maisons et tout, sur une île inconnue de leurs conquérants.» (Bibl. Hist. V, 20).

Cluvier a recours à ce récit qu'il cite longuement pour montrer que les Anciens ont pu connaître l'Amérique (Introd. VI, 11). Mais a-t-il lu Diodore dans le texte? Ce n'est pas impossible, mais peu probable. On sait en effet que l'édition du texte grec par H. Estienne (Genève 1559, édition princeps) a été précédée par des traductions partielles en latin ou en français. Les livres I à V notamment, traduits en latin par

38 Cf. Diodore de Sicile, The Library, éd. et trad. C.H. OLDFATHER, Londres-Cambridge, Loeb Library, 1. II, 1933 (repr. 1979) ; l. V, 1939, (repr. 1993).

39 La description de ces eldorados plus ou moins mythiques est assez fréquente chez les auteurs anciens. Mais les Grecs situent plutôt ces pays vers l'Orient, en des endroits théoriquement accessibles. Strabon par exemple décrit l'Albanie, sur la rive ouest de la mer Caspienne et au sud du Caucase, comme une terre au sol si fertile qu'il produit spontanément toutes sortes de fruits: «les hommes sont exceptionnellement beaux et grands, simples dans leurs manières et dépourvus de tout esprit mercantile» (Géogr. XI, 4, 3-4). L'Hyrcanie de même, sur le bord méridional de la mer Caspienne, est présentée comme extrêmement fertile: "un plant de vigne produit un métrète (sc. 40 litres) de vin, le blé repousse du grain tombé de l'épi, les abeilles édifient leurs ruches dans les arbres et le miel ruisselle des feuilles.» (Géogr. XI, 7, 2). Mais Diodore de Sicile, fidèle sujet de l'empire Romain, préfere situer son Eldorado en plein Océan, hors d'atteinte des hommes. 
Poggio Bracciolini, ont été publiés à Bologne en 1472, tandis que la traduction latine de l'ensemble de l'œuvre, faite par différents auteurs (les livres I à V par Poggio Bracciolini), parut à Bâle en 1548. La traduction latine était probablement plus aisée à trouver et à utiliser que le texte grec.

Vossius, qui définit deux continents nouveaux, l'Amérique ou Inde occidentale, et la Magellanique ou terre Australe (De Philologia XI, 27), assure que beaucoup d'érudits "pensent que regarde l'Australe ce que l'on peut lire de l'itinéraire de Iambulos chez Diodore de Sicile au livre II». Ce Iambulos, parti vers l'Arabie en quête d'épices, fut fait prisonnier par des brigands, puis par des Éthiopiens qui, à partir de la côte d'Éthiopie, les expédièrent par mer, lui et ses compagnons, en direction du sud avec six mois de vivres, à la recherche d'une île heureuse. Ils la trouvèrent au terme de quatre mois de tempêtes: elle était ronde, de 5000 stades de tour, de climat tempéré bien que située sous l'équateur: «les fruits y mûrissent toute l'année» (Bibl. Hist. II, 56). Les habitants, beaux, vigoureux, de grande taille ${ }^{40}$, menaient une vie simple, bien organisée politiquement et moralement, et vivaient cent cinquante ans sans maladie. Iambulos, après y avoir séjourné sept ans, en fut chassé, ainsi que son compagnon, pour avoir contracté de mauvaises habitudes. Quatre mois de navigation difficile les amenèrent en Inde où ils échouèrent sur un marais. Le compagnon y mourut. Iambulos fut d'abord conduit au roi du pays résidant à Palibothra ${ }^{41}$; puis il revint en Grèce par la Perse et rédigea le récit de ses aventures (Bibl. Hist. II, 55-60).

Cet Eldorado situé sous l'équateur, décrit par Diodore, est-il une préfiguration de la terre australe inconnue, que les cartes des $\mathrm{XVI}^{\mathrm{e}}$ et XVII e siècles ${ }^{42}$ représentaient avec des contours très imprécis, et qui s'étendait jusqu'au pôle sud? C'est moins que probable, mais les érudits dont parle Vossius n'hésitaient pas à faire feu de tout bois.

\section{Ps. Aristote, le De Mundo, le De Mirabilibus Auscultationibus}

Un autre texte auquel il est fait souvent référence, c'est le De Mundo, que l'on hésitait alors à attribuer à Aristote ${ }^{43}$, proposant aussi Théophraste comme auteur possible.

40 Diodore ajoute (II, 56) qu'ils avaient la langue divisée en deux parties, si bien qu'ils pouvaient converser avec deux hommes à la fois.

41 Palibothra, capitale de l'Inde du Nord, est l'actuelle Patna.

42 Cf. par exemple la carte de la Mer Pacifique, établie par Abraham Ortelius en 1589, et incluse dans son Theatrum orbis terrarum, Anvers, 1592, où la "Terra australis sive Magellanica nondum detecta» couvre toute la partie sud de la Mer Pacifique. De même sur la carte circulaire catalane de Modène, datée de 1450, la plus grande partie de la moitié sud est occupée par la demi-lune d'un continent inconnu, "terra australis incognita".

43 Cf. Ps. Aristote, $D u$ Monde, trad. et notes J. TRICOT, Paris, Vrin, 1990. Mais G.R EALE et A.P. BOS, Il Trattato Sul Cosmo per Alessandro attribuito ad Aristotele (Milan, Vita e Pensiero, 1974, repr. 1995) soutiennent que ce traité est vraiment d'Aristote. 
On pense aujourd'hui que le rédacteur de ce petit traité serait un péripatéticien fortement influencé par le stoïcisme, peut-être un disciple proche ou lointain de Poseidonios. Ce philosophe célèbre ${ }^{44}$, très intéressé par les questions scientifiques, aurait composé entre autres deux traités (perdus), Éléments de Météorologie, et Du Monde, dont se serait inspiré, sans doute au Irr siècle de notre ère, l'auteur de l'actuel De Mundo.

Au chapitre III, est évoquée l'existence probable d'autres terres, en dehors du monde alors connu: "Le langage courant a divisé la terre habitée en îles et continents, ignorant que la terre n'est tout entière qu'une seule île, que baigne à l'entour la mer Atlantique, comme on la nomme. Mais il est vraisemblable qu'il existe plusieurs autres continents séparés du nôtre et situés de l'autre côté de l'eau, les uns plus grands que celui-ci, les autres plus petits, mais tous, à l'exception du nôtre, invisibles à nos yeux. En effet, ce que sont les îles de notre monde par rapport à nos mers, le monde habité l'est par rapport à la mer Atlantique, et beaucoup d'autres continents à l'égard de la mer prise dans son ensemble, car ils sont comme de très grandes îles baignées par d'immenses mers qui les entourent.» (392 b). On lit un peu plus loin: «Parmi les îles, les unes sont de grandes îles, comme l'ensemble de ce qu'on a appelé la terre habitée,mais il y en a beaucoup d'autres, baignées tout autour par des mers immenses.» (393 a).

Cluvier qui cite ce traité l'a lu non pas dans le texte mais dans la traduction latine, élaborée par Apulée ${ }^{45}$ (c. 125-170), qui en a largement diffusé la teneur. Au reste, outre cette traduction d'Apulée, contenue dans ses Euvres Complètes dont l'édition princeps fut publiée à Rome en 1469 et qui jouirent d'une dizaine d'éditions jusque vers 1600 (dont une procurée par Scaliger à Leyde en 1600 précisément), il y eut deux autres traductions latines 46 faites à partir du grec au XIII siècle, l'une par

44 Pour Poseidonios de Rhodes, ou d'Apamée (c. 135-50), dont toute l'œuvre est perdue, cf. K. REINHARDT, Poseidonios, Munich, Beck, 1921, ou M.L AFRANQUE, Poseidonios, essai de mise au point, Paris, P.U.F., 1964. Et aussi, Posidonius, I, The Fragments, II, The Commentary, L. EDELSTEIN and I.G. KIDD, Cambridge, University Press, 1972 et 1988.

45 Cf. Apulée, Opuscules Philosophiques (Du Dieu de Socrate, Platon et sa doctrine, Du Monde) et fragments, éd. et trad. J. BEAUJEU, Paris, C.U.F., 1973. Dans le passage auquel Cluvier fait allusion, Apulée disait: "Je n'ignore pas que la plupart des spécialistes ont divisé ainsi les terres émergées: pour une part, selon eux, ce sont des îles; l'autre part, ils l'ont appelée continent, sans savoir que toute l'immensité de notre continent est entourée par l'Océan Atlantique et ne forme elle-même qu'une île avec toutes ses îles. En effet il y en a de semblables à celles-ci, les unes plus grandes, les autres plus petites, qu'entoure l'Océan; mais elles passent à juste titre pour inconnues puisque même celle-ci que nous habitons, nous ne pouvons la parcourir tout entière. Car de même que les flots séparent ces îles proches qui se trouvent dans notre mer, de même les îles lointaines situées dans l'Océan universel sont environnées par des étendues d'eau plus vastes.» (392b-393a).

46 Cf. L. MiNiO-PALUEllo, Opuscula - The Latin Aristotle, Amsterdam, Hakkert, 1972, p.108-113. 
Nicolo Siculo, qui fut imprimée à Venise en 1496, l'autre par Bartholomée de Messine, qui resta sous forme manuscrite. Aux XVe et XVIe siècles, six ou sept traductions latines de ce traité faussement attribué à Aristote furent effectuées, et publiées (comme celle de Guillaume Budé, Paris, 1526), ou simplement conservées en manuscrit (comme celle de Jean Argyropoulos, faite vers 1471).

Vossius, qui ne cite pas le De Mundo, évoque en revanche un autre traité pseudoaristotélicien, compilation tardive faite entre le $\mathrm{II}^{\mathrm{e}}$ et le $\mathrm{VI}^{\mathrm{e}}$ siècles, le De mirabilibus auscultationibus qu'il a dû lire dans le texte ${ }^{47}$ : l'édition princeps d'Aristote le contenant avait paru chez Alde en 1495-1498. Mais la traduction latine, faite vers 1260 par Bartholomée de Messine qui s'était spécialisé dans les Pseudepigrapha, avait déjà été publiée à Venise en 1482, 1483, 1489, et le fut à Bâle en 1538. Voici le texte auquel Vossius fait allusion, proche parent de celui de Diodore de Sicile, au livre V: «Dans la mer au delà des Colonnes d'Hercule, on dit que les Carthaginois ont découvert une île déserte, portant des arbres de toutes sortes et des fleuves navigables, et admirable par toutes sortes de fruits, distante de plusieurs jours de navigation. Les Carthaginois la fréquentèrent souvent vu sa prospérité, certains même y élirent domicile; mais les chefs des Carthaginois annoncèrent qu'ils puniraient de mort ceux qui se rendraient en bateau dans cette île, et qu'ils en massacreraient tous les habitants afin qu'ils n'aillent pas raconter des histoires; ils craignaient qu'une masse des leurs ne se rassemble sur cette île pour s'en emparer, ce qui ruinerait la prospérité des Carthaginois.» (De mirabilibus auscultationibus 84, 336b). Vossius ajoute que, pour beaucoup, l'île ainsi décrite serait l'Amérique; tel aurait été l'avis ${ }^{48}$ de Jean Goropius Becan, au livre III de ses Origines d'Anvers, publiées à Anvers en 1569, d'Adrien Turnèbe, au livre XX, chap. 11, de ses Adversaria (Paris, 1580) ainsi que de Jacques de Pamèle, dans ses Argumenta et annotationes in Tertulliani opera (Paris, 1635). L'accumulation de ces références si diverses montre bien que la question des relations entre le Nouveau Monde et le savoir des Anciens n'a pas occupé que les géographes.

Quant au véritable Aristote, on lui reprochait en général d'avoir répandu l'idée que la zone située entre les tropiques était "torride» et donc inhabitable par suite de la chaleur. Comme beaucoup d'Anciens en effet, adeptes de la géométrie de la sphère, il considérait que sur le globe terrestre seule la zone tempérée, entre tropique et cercle

47 Cf. Aristotle, t. XIV, transl. W.S. HETT, Londres-Cambridge, Loeb Library, 1980. Montaigne lui aussi faisait appel, on l'a vu (cf. supra, p. 172), au témoignage de ce traité d'Aristote, «au moins si ce petit livre est à lui».

48 Il semble que Vossius se soit cherché ici des garants. Jean Becan (ou Goropius Becanus), né en 1518 dans le Brabant, se fixa par la suite à Anvers où il mourut en 1572; il rédigea les Origines d'Anvers en neuf livres dont le troisième avait pour titre Niloscopium. Le Français Adrien Turnèbe (1512- 1565) occupa à partir de 1547 la chaire de grec puis de philosophie grecque et latine à Paris, où il eut pour élève $H$. Estienne; les Adversaria, en trois parties, réunissaient des notes sur les auteurs anciens, parcourus dans le désordre (Strasbourg, 1604). Jacques de Pamèle (1536-1587), né à Bruges, étudia la philosophie à Louvain puis Paris; il édita Cassiodore, Cyprien et Tertullien (Paris, 1584). 
arctique $^{49}$, était habitable, les zones extrêmes, les deux glaciales et la zone torride étant inhabitables par suite du froid ou de la chaleur ${ }^{50}$. Or la partie du Nouveau Monde que l'on connaissait le mieux, notamment le Pérou, le Brésil, le Mexique, était majoritairement située entre les tropiques, dans ce que les Anciens et Aristote appelaient la zone torride jugée inhabitable. Pour avoir passé de longues années au Pérou et au Mexique, José de Acosta avait montré et proclamé que ces territoires jouissaient d'un climat relativement tempéré, très agréable en tout cas, et étaient donc particulièrement propices à l'habitation humaine. Si l'on voulait suggérer que les Anciens avaient une certaine connaissance, même imparfaite, de l'Amérique, il fallait trouver dans leur littérature des preuves qu'ils savaient la zone torride habitable, et habitée. Or des preuves en ce sens, on pouvait en trouver en particulier chez Pline l'Ancien.

\section{Pline l'Ancien (23-79), l'Histoire Naturelle}

À Pline l'Ancien, auteur d'une Histoire Naturelle en trente six livres, immense encyclopédie qui dispensait les lecteurs d'avoir recours directement aux sources grecques, José de Acosta emprunte d'abord le récit des périples autour de l'Afrique, qui ne pouvaient se faire qu'en traversant ladite zone torride, et puis ce qui est dit de Taprobane.

C'est d'abord le Carthaginois Hannon qui, avant 480 av. J.-C., aurait navigué depuis Gadès jusqu'en Arabie ${ }^{51}$. Plus tard, vers 110 av. J.-C., Eudoxe de Cyzique aurait fait le tour de l'Afrique dans l'autre sens. Voici ce que rapporte Pline au livre

49 Le cercle arctique en question n'est pas notre cercle polaire, mais le parallèle $54^{\circ} \mathrm{N}$ (ou S), qui en fait se trouvait être le cercle de perpétuelle visibilité pour l'horizon de Rhodes $\left(36^{\circ} \mathrm{N}\right)$.

50 Cf. Météorologiques II, 5, 362 a 33-b 10: «Il y a deux secteurs habitables à la surface de la terre, l'un dans lequel nous habitons, vers le pôle supérieur, l'autre vers l'autre pôle, le pôle sud... Ce sont les seules régions habitables. Les régions au delà des tropiques ne sont pas habitables, car l'ombre ne s'y dirige pas vers le nord, et nous savons que la terre cesse d'être habitable avant que l'ombre disparaisse ou se dirige vers le sud, tandis que les régions situées sous l'Ourse sont inhabitables par suite du froid.» En fait Aristote, après avoir indiqué que sont habitables les deux zones tempérées, nord et sud, ne considère par la suite que les limites de la zone comprise dans l'hémisphère nord, laissant au lecteur le soin d'extrapoler pour l'hémisphère sud. José de Acosta avait cité, dans ce même chapitre des Météorologiques, le passage précisant que, "si l'immensité de la mer ne l'empêchait, l'on pourrait faire le tour de la terre en longitude.» (362 b 18).

51 Sur le périple d'Hannon, cf. J. DESANGES, "Les routes africaines de l'aventure, rêve et réalité", in Les routes millénaires, Paris, Nathan, 1988, p. 65-86. Hérodote avait également relaté (Enquête IV, 42) le périple des marins de Néchao autour de l'Afrique: «Et ils racontaient - chose que, quant à moi, je ne crois pas, mais que d'autres peuvent croire - que, pendant qu'ils accomplissaient le périple de Libye, ils avaient eu le soleil à leur droite.» 
II ${ }^{52}$ : «Alors que la puissance de Carthage était florissante, Hannon fit le grand tour depuis Gadès jusqu'aux frontières d'Arabie et publia le récit de son périple, comme le fit aussi Himilcon que l'on envoya dans le même temps reconnaître les abords extérieurs de l'Europe. En outre Cornelius Népos garantit que de son temps un certain Eudoxe 53, fuyant le roi Ptolémée Lathyre (c. 141-81), sortit du golfe d'Arabie et fit voile jusqu'à Gadès.» (Hist. Nat. II, 169). Ainsi la circumnavigation de l'Afrique, comportant un long séjour dans la zone torride, aurait eu lieu au moins deux fois, mais probablement bien plus souvent. De quoi montrer que les Anciens ont su, par la pratique, que dans la zone située entre les tropiques la vie était possible, contrairement à ce que soutenaient les théoriciens comme Aristote.

Autre emprunt que fait d'Acosta à Pline ${ }^{54}$, et que d'autres feront, pour montrer que les Anciens savaient une partie au moins de la zone torride habitable, la description de l'île de Taprobane (Hist. Nat. VI, 81-91), que d'Acosta identifie à Sumatra, alors que l'on a l'habitude d'y voir l'île de Ceylan. " (81) Taprobane a été longtemps considérée comme un autre monde, sous le nom de terre des Antichthones 55. C'est l'époque d'Alexandre le Grand et ses entreprises qui ont prouvé manifestement que c'était une île. Onésicrite son amiral a écrit que les éléphants y sont plus grands et plus belliqueux qu'en Inde. Mégasthène ${ }^{56}$, qu'elle est partagée par un fleuve, que les habitants sont appelés Palaeogones et qu'ils produisent plus d'or et de grosses perles que les Indiens. Ératosthène 57 en a donné les dimensions: 7000 stades de long ( $s c$. dans les $1100 \mathrm{~km}$ ) et 5000 de large ( $s c$. dans les $780 \mathrm{~km}$ ), et indiqué qu'elle n'a pas de villes mais 750 villages. (82) Elle commence à la mer orientale et s'étend face à l'Inde dans le sens Est-Ouest; on la croyait jadis à vingt jours de navigation de la nation des Prases (sc. des riverains du Gange)... mais la distance fut évaluée plus tard à sept jours d'après la vitesse de nos navires [...]. (84)

52 Pline l'Ancien, Histoire Naturelle II, éd. et trad. J. BEAUJEU, Paris, C.U.F., 1950.

53 Il s'agit d'Eudoxe de Cyzique, dont l'histoire est racontée avec force détails par Strabon (Géogr. II ,3, 4) sur la foi de Poseidonios. C'est également de Poseidonios que la tient Cornelius Népos, cité par Pline et aussi par Pomponius Mela, Chorographie III, 9, 90.

54 Pline l'Ancien, Histoire Naturelle VI, éd. J. ANDRÉ et trad. J. FILIOZAT, Paris, C.U.F., 1980.

55 Dans la conception traditionnelle, d'un globe terrestre abritant peut-être quatre mondes habités dans les quatre quarts du globe, dont un seul, le nôtre, serait connu, les Antichthones seraient les habitants du monde situé dans l'hémisphère sud, au dessous du monde connu, tandis que les antipodes occuperaient l'autre quart du même hémisphère sud.

56 Mégasthène ( $f l .300$ av. J.-C.) fut ambassadeur de Séleucos Nicator auprès du roi Maurya Sandracottos à Palibothra (sc. Patna). Il composa des Indica en quatre livres, très utilisés par les géographes postérieurs.

57 Ératosthène de Cyrène (c. 275-195), l'inventeur du terme geographia et le premier auteur d'un traité portant ce titre, dressa aussi la première carte scientifique du monde habité fondée sur sa mesure de la circonférence terrestre obtenue par moyens gnomoniques. Cf. G.A UJAC, Ératosthène de Cyrène, le pionnier de la géographie, Paris, éd. du C.T.H.S., 2001. 
Nous avons parlé jusqu'ici d'après les auteurs anciens. Des renseignements plus exacts nous sont parvenus sous le principat de Claude (regn. 41-54), et même des ambassadeurs sont venus de cette île [...]. (87) Ils admiraient chez nous la Grande Ourse et les Pléiades comme en un ciel nouveau, et déclaraient que même la lune n'était visible chez eux que du huitième au seizième jour et que dans leurs nuits brillait Canopus, une très grande et brillante étoile. Mais ce qui les étonnait le plus était que leurs ombres tombaient du côté de notre ciel et non du leur, et que le soleil se levait à gauche et se couchait à droite au lieu du contraire». Autant d'indications qui plaçaient Taprobane au sud du tropique d'été, prouvant que la zone torride était habitable, au moins partiellement.

\section{Sénèque (4 av.-65 ap. J.-C.), Médée}

Autre argument fréquemment cité: quelques vers de la Médée de Sénèque, le philosophe stoïcien, précepteur de Néron, auteur de dialogues, de traités philosophiques, des fameuses Lettres à Lucilius, mais aussi de tragédies, comme Médée ${ }^{58}$, composée vers 63-64, soit très peu de temps avant sa mort programmée par Néron. En 62, il avait commencé à travailler aux Questions Naturelles, où il abordait toutes sortes de problèmes scientifiques 59 .

C'est donc fort de sa confiance dans les progrès incessants de la science qu'il fait dire au Chœur, dans Médée ${ }^{60}$, les vers dont ceux qui se rapportent à un futur lointain seront jugés prophétiques par les historiens du Nouveau Monde: «Maintenant la mer est soumise et se plie à toutes nos lois: il n'est nul besoin de l'illustre Argo 61 assemblée par la main de Pallas et sur laquelle les rameurs sont des rois: la première barque venue s'aventure en haute mer; toutes les limites se trouvent reculées et les

58 Corneille s'en est inspiré pour sa propre Médée.

59 Cf. par exemple, Questions Naturelles VII, II, 3: "Est-ce le monde qui tourne autour de la terre immobile ou le monde est-il fixe et la terre roule-t-elle dans l'espace? En effet des savants ont affirmé que l'univers nous emporte sans que nous nous en doutions; dès lors les levers et les couchers ne sont pas les effets du mouvement du ciel, c'est la terre qui se lève et qui se couche. Voilà une question digne que nous l'examinions. Car il s'agit de savoir quelle est notre situation dans le monde, si nous avons en partage la demeure la plus paresseuse ou la plus rapide, si Dieu fait rouler l'univers autour de nous, ou si c'est nous qu'il mène." (trad. P. OLTRAMARE, Paris, C.U.F., 1929). Allusions aux hypothèses révolutionnaires d'Héraclide de Pont (c. 390-310) et d'Aristarque de Samos (c. 310-230), qui plaçaient le soleil au centre et faisaient tourner la terre autour de lui.

60 Cf. Sénèque, Tragédies, t. I, Hercule furieux, les Troyennes, les Phéniciennes, Médée, éd. et trad. F.R. Chaumartin, Paris, C.U.F., 1996; ou Sénèque, Médée, trad. Ch.G UITTARD, Paris, Flammarion, 1997, dont j'emprunte la traduction.

61 Allusion à l'expédition des Argonautes vers la Colchide, sur la rive orientale de la mer Noire, en quête de la Toison d'or; ils s'en empareront avec l'aide de Médée, la fille du roi de Colchide, amoureuse de Jason. Le récit légendaire de l'expédition avait été chanté en grec par Apollonios de Rhodes, au III s. av. J.-C., et en latin par Valerius Flaccus (c.45-88 ap. J.-C.). 
villes ont dressé leurs murailles sur de nouvelles terres [...]. Plus tard, dans le cours des années, viendront des temps où l'Océan relâchera son emprise sur le monde, où la terre s'ouvrira dans son immensité, où Téthys nous révèlera de nouveaux mondes et où Thulé ${ }^{62}$ ne sera plus la limite de l'univers.» (v. 364-379, trad. Ch. Guittard).

José de Acosta commente: «Beaucoup ${ }^{63}$ pensent que Sénèque le tragique fut le prophète de ces Indes Occidentales dans Médée» (Hist. I, 11). Botero, qui apprécie la valeur prophétique de ces vers, reproche à l'auteur d'avoir mentionné Thulé comme limite du monde habité, ce qui suggèrerait une prolongation du domaine connu vers le Nord. Or c'est vers l'Ouest qu'a eu lieu l'extension, par la découverte du Nouveau Monde. Et donc, argumente Botero, Sénèque aurait dû parler de Cadix, et non pas de Thulé, pour que cette prophétie prenne tout son sens.

\section{Festus Aviénus (IV siècle), Ora Maritima}

Vossius, pour montrer que les Anciens avaient l'habitude de naviguer au-delà des colonnes d'Hercule, invoque Festus Aviénus ${ }^{64}$, dans les Ora Maritima. Ce poème en vers iambiques décrit surtout la côte, de Marseille à Gadès-Cadix, mais rappelle aussi bien des voyages effectués par les Grecs ou les Carthaginois dans l'Océan Atlantique, vers 500 av. J.-C. Vossius cite un fragment du poème dans le De Philologia (XI, 27), puis rappelle cette citation dans le De Scientiis Mathematicis (XLII, 10).

Après avoir évoqué les mystérieuses îles Estrymnides ${ }^{65}$, «aux larges plaines et aux riches mines d'étain et de plomb», puis Albion et l'Irlande, Aviénus continuait par ces vers qui ont frappé Vossius: «Les Tartessiens ${ }^{66}$ avaient l'habitude de commercer aux limites des Estrymnides: de même les colons de Carthage et les gens répandus autour des Colonnes d'Hercule visitaient ces régions. Le Carthaginois

62 Thulé était l'île mystérieuse, dont avait entendu parler Pythéas le Massaliote, dans son périple autour de l'Europe du Nord-Ouest: le jour du solstice d'été, le soleil ne se couchait pas; Thulé était donc sur le cercle polaire, à $66^{\circ} \mathrm{N}$. Pour Pythéas et Ératosthène, le parallèle de Thulé constituait la limite septentrionale du monde habité. D'où l'Ultima Thule de Virgile.

63 Parmi eux, se trouvait Lopez de Gomara, comme il a été signalé plus haut (p. 164).

64 Cf. Festus Avienus, Ora Maritima, ed. et trad. A. BERTHELOT, Paris, librarie anc. H.Champion, 1934. Avienus est aussi l'auteur d'une Description du monde, en quelque 1400 hexamètres s'inspirant beaucoup du poème grec de Denys le Périégète ( $f l .130$.

65 ap.J.-C.).

Ces îles où l'on se procurait de l'étain étaient appelées par les Grecs Cassitérides. On les situait soit vers l'archipel des Sorlingues, devant la Cornouaille anglaise, soit au large du cap Finisterre, pointe extrême au Nord-Ouest de l'Ibérie. On hésitait d'autant plus sur leur localisation que le trajet vers ces îles avait été longtemps tenu secret par les commerçants phéniciens ou carthaginois désireux de conserver le monopole sur ce produit précieux et rare. Cf. Strabon, Géogr. III, 5, 11.

66 Tartessos, proche de l'embouchure du Guadalquivir, avait été occupée par les Phéniciens, hardis marins et grands commerçants, qui importaient l'étain de Grande-Bretagne. 
Himilcon ${ }^{67}$, qui rapporte avoir lui-même expérimenté cette navigation, affirme que c'est à peine si l'on peut les atteindre en quatre mois. Ainsi nul souffle ne propulse le navire, l'eau de cette mer paresseuse semble engourdie. Il ajoute que du fond montent une multitude d'algues qui souvent retiennent le bateau comme une haie; néanmoins, dit-il, la mer est sans profondeur, à peine une mince couche d'eau recouvre le sol; toujours des animaux marins circulent ça et là, des monstres nagent entre les navires qui se traînent, lents et inertes.» (v. 113-129, trad. A. Berthelot).

Citant ces vers, Vossius prétendait avec optimisme: «ils paraissent s'appliquer à l'Amérique», ce qui est loin d'être évident, puisque le périple d'Himilcon, tel que le présente Aviénus, se serait surtout effectué en direction du nord, au large des côtes européennes de l'Atlantique.

Tels sont les auteurs principaux invoqués au cours des discussions qui opposaient les tenants des thèses opposées sur la connaissance que les Anciens avaient pu avoir de ce Nouveau Monde récemment découvert. Ce sont en majeure partie des auteurs latins plus ou moins tardifs (mais on évoquait aussi à l'occasion les poètes Virgile ou Ovide); les quelques auteurs grecs cités semblent l'avoir été le plus souvent à travers des traductions latines.

Or, si l'on voulait montrer que les Anciens ont, sinon connu l'Amérique, du moins subodoré l'existence d'un continent ou de plusieurs continents inconnus, il existait nombre de textes grecs, à teneur plus ou moins scientifique, qui auraient fourni, sur bien des sujets de la controverse, des arguments de poids.

\section{D’autres textes utiles, mais non utilisés}

Les grands thèmes abordés dans ces débats portaient essentiellement d'abord sur la possibilité pour les anciens d'envisager d'autres mondes habités, en dehors de celui qu'ils occupaient, puis sur leur ignorance supposée de l'hémisphère austral, enfin sur la croyance en l'inhospitalité pour les humains de la zone dite torride, entre les tropiques. Si ces thèmes avaient déjà fait dans l'Antiquité l'objet de maintes discussions, plus ou moins passionnées, le monde savant avait, lui, sur bien des points, des opinions relativement justes, dont poètes et écrivains, grecs ou latins, se sont faits souvent les échos.

67 La reconnaissance des côtes atlantiques de l'Europe par Himilcon eut lieu vers 500 av. J.-C., mais ce qui en est dit ici rend difficile l'identification de son itinéraire. 


\section{Les autres «mondes habités» possibles}

Les géographes grecs en général - Claude Ptolémée 68 étant une exception tardive ont considéré que «notre» monde habité, soit les trois continents connus, EuropeAsie-Afrique, était situé au nord de l'équateur, dans un des quarts du globe terrestre. Les trois autres quarts, encore inconnus, vu l'impossibilité de s'aventurer très loin des côtes, pouvaient donc être occupés par des terres, peuplées ou non, ou par de vastes mers.

Ératosthène de Cyrène ${ }^{69}$, qui avait le premier, au III ${ }^{\mathrm{e}}$ s. av. J.-C., mesuré de façon scientifique la circonférence terrestre, attribuait à la longueur du monde connu, d'Est en Ouest, le long du parallèle de Rhodes $\left(36^{\circ} \mathrm{N}\right)$ considéré comme le parallèle central de la carte, un peu plus du tiers du parallèle entier. D'où il concluait que, «si l'immensité de l'océan Atlantique n'y faisait obstacle, il nous serait possible d'aller par mer d'Ibérie jusqu'en Inde: il suffirait de suivre le même parallèle et de parcourir la section qui reste, une fois ôtée la distance définie ci-dessus, soit un peu plus du tiers de la circonférence totale, en admettant une valeur inférieure à 200000 stades pour le parallèle d'Athènes ${ }^{70}$ sur lequel a été faite la précédente répartition en stades depuis l'Inde jusqu'à l'Ibérie.» (Strabon, Géogr. I, 4, 6). Si Strabon ${ }^{71}$, qui transmet l'enseignement d'Ératosthène, reconnaît que le raisonnement est correct du point de vue mathématique, il émet des doutes sur la réalisation d'un tel projet, car «l'on admet que, dans la même zone tempérée, il peut y avoir deux mondes habités ou plus, et surtout à la hauteur du parallèle d'Athènes, dans la partie qu'il décrit à travers l'Océan Atlantique» (Géogr. I, 4, 6). La possibilité d'un autre continent, habité ou non, dans la zone tempérée nord était donc communément envisagée.

Vers le milieu du IIe s. av. J.-C. en effet, le stoïcien Cratès de Mallos, qui fut directeur de la Bibliothèque de Pergame, s'était rendu célèbre pour avoir fabriqué un globe terrestre de trois mètres de diamètre (cf. Strabon, Géogr. II, 5, 10) sur lequel il avait représenté quatre mondes habités symétriques, séparés par des ceintures

68 Sur ce géographe célèbre, cf. par exemple, G. AUJAC, Claude Ptolémée, astronome, astrologue, géographe, Paris, éd. du C.T.H.S., 1993 (repr. 1998).

69 Sur Ératosthène de Cyrène (c. 275-195), qui fut à la tête de la Bibliothèque d'Alexandrie pendant une cinquantaine d'années, cf. n. 57.

70 Ératosthène avait évalué à 252000 stades la circonférence terrestre, ce qui mettait le degré de méridien à 700 stades. Les parallèles à la surface du globe terrestre étant tous inférieurs à l'équateur, à proportion de leur éloignement, le parallèle de Rhodes (ou d'Athènes), à $36^{\circ} \mathrm{N}$, était évalué à 200000 stades en chiffres ronds: le degré de longitude sur le parallèle de Rhodes valait alors dans les 555 stades. La longueur du monde habité, de l'Ibérie jusqu'en Inde, avait été fixée par Ératosthène à quelque 78000 stades (soit dans les $140^{\circ}$ de longitude). On a beaucoup discuté sur les diverses valeurs du

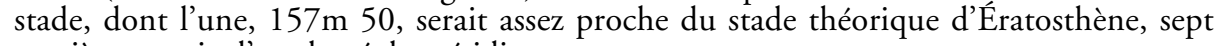
centième partie d'un degré de méridien.

71 Sur Strabon (c. 63 av.- 24 ap. J.-C.), contemporain de l'empereur Auguste, cf. G.A UJAC, Strabon et la science de son temps, Paris, Les Belles Lettres, 1966. Et aussi et surtout Strabon, Géographie, t. I (l. I et II), éd. et trad. G. AUJAC, Paris, C.U.F., 1968. 
océaniques déployées l'une le long d'un méridien et l'autre le long de l'équateur; de ces quatre mondes habités, un seul était connu, «le nôtre», les barrières océaniques empêchant que l'on ne débarque sur les autres. Voilà en tout cas une belle anticipation des expéditions futures, quand les navigateurs, connaissant l'usage de la boussole, auront la hardiesse de traverser ces océans, réputés infranchissables par les Anciens.

Voulant rivaliser avec Ératosthène et sa mesure du globe terrestre, Poseidonios ${ }^{72}$, utilisant un autre procédé, astronomique, qui se révélait bien peu fiable, réduisait à 180000 stades la circonférence terrestre, ce qui rendait encore plus courte la distance à parcourir sur mer, d'Ibérie en Inde (il avait également réduit la longueur du monde habité): «Poseidonios émet l'hypothèse que les quelque 70000 stades qui représentent la longueur du monde habité valent la moitié du cercle entier sur lequel est prise cette longueur, de sorte que, dit-il, si, partant de l'Occident, l'on naviguait par vent d'est, au bout d'un nombre égal de stades, on aboutirait aux Indes.» (Strabon, Géogr. II, 3, 6).

Or c'est sur cette mesure (largement erronée) de la circonférence terrestre que se sont fondés Marin de Tyr ${ }^{73}$ et Ptolémée. De plus, Marin de Tyr attribuait $225^{\circ}$ de longitude à la longueur du continent eurasiatique, qui n'en vaut en fait que 140 , du cap Vert à Pékin, ou de Gadès-Cadix au Japon. Ptolémée, probablement pour simplifier le tracé de la carte, réduisait à $180^{\circ}$ l'extension du continent eurasiatique, mais ne le limitait pas, à l'Extrême Orient, par la mer, ce qui laissait supposer une continuation des terres au delà des $180^{\circ}$. L'un comme l'autre ont donné de faux espoirs (mais beaucoup de courage) à Christophe Colomb, qui possédait un exemplaire de la Géographie de Ptolémée en latin (elle avait été traduite dès 1409), dans l'édition de Rome de 1478. Porté par l'espérance, Colomb est parti à la recherche des Indes.

\section{L'hémisphère austral}

La géométrie de la sphère ${ }^{74}$ avait appris depuis longtemps aux mathématiciens ou géographes grecs que le globe terrestre, réplique de la sphère céleste, comprenait deux hémisphères, l'un septentrional, l'autre méridional, séparés par l'équateur. Les zones

72 Sur Poseidonios, cf. n. 44. Les procédés utilisés par Ératosthène et par Poseidonios pour mesurer la circonférence terrestre ont été décrits au $\mathrm{II}^{\mathrm{e}}$ s. ap. J.-C. par Cléomède, De motu circulari I, 10 (ou Théorie élémentaire, trad. R. GOULET, Paris, Vrin, 1980, p. 121126).

73 Marin de Tyr, légèrement antérieur à Ptolémée, avait voulu corriger la carte du monde habité procurée par Ératosthène, mais il en était resté à des corrections occasionnelles, ce qui a donné à Ptolémée le désir d'opérer une réfection complète de la carte, sur nouveaux frais.

74 Sur les enseignements de la géométrie de la sphère, cf. G. AUJAC, La sphère, instrument au service de la découverte du monde, Caen, Paradigme, 1993. 
étaient elles aussi symétriques par rapport à l'équateur; dans l'hémisphère austral, il y avait donc une zone tempérée et une zone glaciale, équivalentes à celles de l'hémisphère nord. Strabon est très clair dans cet exposé qui présente l'essentiel des connaissances (théoriques) de l'époque. "Il faut poser en préalable que le ciel a cinq zones, cinq zones aussi la terre, et que les zones portent le même nom ici-bas qu'en haut [...] Les zones seraient délimitées par des cercles parallèles à l'équateur, tracés de chaque côté de celui-ci, deux d'entre eux isolant la zone torride, deux autres à la suite formant à partir de la zone torride les deux zones tempérées, et à partir des zones tempérées les zones glaciales... Étant donné que l'équateur coupe en deux la totalité du ciel, nécessairement la terre est aussi divisée en deux par l'équateur terrestre. Chacun des hémisphères, au ciel comme sur terre, s'appelle l'un boréal, l'autre austral; aussi puisque la zone torride est divisée en deux par le même cercle, une partie en sera boréale, l'autre australe. Il est clair également que, des deux zones tempérées, l'une sera boréale, l'autre australe, du nom de l'hémisphère dans lequel elle se trouve. On appelle hémisphère boréal celui qui contient la zone tempérée dans laquelle, en regardant d'Est en Ouest, on a le pôle à sa droite, l'équateur à sa gauche, ou encore celui dans lequel, quand on regarde vers le midi, on a le couchant à sa droite, le levant à sa gauche; pour l'hémisphère austral, c'est l'inverse.» (Strabon, Géogr. II, 5, 3).

Comme l'on pensait aussi que seules les zones tempérées étaient susceptibles d'être habitées, il était courant d'imaginer d'autres mondes habités possibles, en dehors du nôtre: on leur donnait, sans les connaître, des noms évocateurs. C'est l'enseignement que dispense Géminos ${ }^{75}$, dans son Introduction aux Phénomènes, manuel élémentaire d'astronomie et de géographie mathématique. Selon sa nomenclature, «sont contigus les lieux situés dans la même moitié de la même zone; sont symétriques les lieux situés dans la même zone, dans l'autre moitié du cercle; sont opposés ${ }^{76}$ ceux situés dans la zone tempérée australe mais dans un même hémisphère; sont antipodes ceux situés dans la zone australe mais dans l'autre hémisphère, diamétralement opposés à notre monde habité, d'où leur nom d'antipodes: tous les graves en effet convergeant vers le centre du fait de l'attraction des corps vers le milieu, si, à partir d'un lieu quelconque situé dans notre monde habité, l'on tire une droite en direction du centre de la terre et si on la prolonge, les lieux situés à l'extrémité du diamètre dans la zone australe sont les antipodes de ceux situés dans la zone boréale» (Introd. aux Phén. XVI, 1-2). Mais ce vulgarisateur intelligent ne manquait pas de mettre en garde ses lecteurs contre une interprétation trop littérale de cet enseignement.. "Quand nous parlons de la zone australe et de ses habitants, en particulier des antipodes qu'elle abrite, il convient de ne pas se

75 Géminos de Rhodes ( $f$ l. 50 av. J.-C.) est l'auteur d'un important traité (perdu) sur La Science mathématique, d'un Abrégé des Météorologiques de Poseidonios (également perdu), et de ce manuel de vulgarisation heureusement conservé. Cf. Géminos, Introduction aux Phénomènes, éd. et trad. G. AUJAC, Paris, C.U.F., 1975.

76 Ce sont les Antichthones dont parlait Pline, à propos de Taprobane (cf. n. 55). 
méprendre sur nos propos: nous n'avons aucune information sur la zone sud, nous ignorons si elle contient ou non des habitants, mais, étant donné le système sphérique d'ensemble, étant donné la forme de la terre et la progression du soleil entre les tropiques, il existe certainement une seconde zone, au sud, qui a le même climat tempéré que celle, au nord, où nous habitons nous-mêmes. De même quand nous parlons d'antipodes, nous n'affirmons pas qu'il existe effectivement des hommes qui nous seraient diamétralement opposés, mais seulement qu'il existe sur la terre un lieu habitable qui nous est diamétralement opposé.» (Introd. aux Phén. XVI, 19-20). On ne saurait être plus prudent.

Nul des quelques auteurs, postérieurs à la découverte du Nouveau Monde, qui ont été retenus pour illustrer cette controverse sur les rapports entre les Anciens et l'Amérique ne cite cette si utile Introduction aux Phénomènes, dont l'édition princeps, avec traduction latine (sous le titre peut-être dissuasif de Elementa astronomiae), fut publiée à Nuremberg en 1590, puis à Lyon en 1603. Le traité de Géminos avait été traduit en arabe probablement vers le IX ${ }^{\mathrm{e}}$ siècle, et de là, traduit en latin par Gérard de Crémone, travaillant à Tolède vers 1170, puis en hébreu par Moses Ibn Tibbon, à Naples, en 1246. Quelques chapitres, dont celui sur les zones, en avaient été publiés en 1499 à Venise par les soins d'Alde Manuce, sous le titre trompeur de Sphère de Proclus, où le texte grec était accompagné d'une traduction en latin procurée par Thomas Linacre. Cela avait peut-être contribué à faire oublier le véritable auteur.

\section{La zone torride habitable? L'équateur tempéré?}

On se souvient que l'un des arguments utilisés par d'Acosta et d'autres pour montrer que les Anciens n'ont pas connu le Nouveau Monde est la conviction, qu'il prête d'ailleurs surtout à Aristote, que la zone torride serait inhabitable, alors que l'on avait fait l'expérience dorénavant qu'entre les tropiques la vie était parfaitement possible et le climat relativement agréable. D'où, pour les défenseurs des Anciens, les références aux récits des diverses circumnavigations de l'Afrique qui prouvaient que, dans le monde antique aussi, on savait que la zone torride n'était pas aussi insupportable que ne le prétendait la théorie. Les humanistes ne citaient que Pline l'Ancien. Mais Poseidonios avant lui avait rappelé «ceux dont la tradition veut qu'ils aient fait le tour de la Libye, évoquant l'opinion d'Hérodote ${ }^{77}$ que des explorateurs à la solde de

77 En fait Hérodote (Enquête IV, 42) parle des Phéniciens partis d'Égypte sur l'ordre du pharaon Néchao, entre 609 et 594, et qui au bout de trois années de périple avaient regagné l'Égypte en passant par le détroit de Gibraltar. 
Darius auraient bouclé le circuit par mer, citant également Héraclide du Pont ${ }^{78}$ qui, dans un de ses dialogues, fait dire à un mage arrivé chez Gélon qu'il vient d'effectuer le circuit par mer... Puis Poseidonios raconte l'histoire d'un certain Eudoxe de Cyzique 79 [...]» (Strabon, Géogr. II, 3, 4). De cet Eudoxe, dont parle également Pline, sur la foi de Cornelius Népos ${ }^{80}$, Poseidonios retraçait avec force détails les démêlés particulièrement rocambolesques avec les souverains d'Égypte, et les diverses tentatives de périples, souvent avortées, mais il reconnaissait honnêtement qu'il ignorait la fin de l'histoire. Or le tour de l'Afrique impliquait la traversée de la zone torride et la preuve que ces lieux étaient habités.

Si ces tentatives de circumnavigation de l'Afrique restaient épisodiques, et furent souvent contestées, les Anciens n'ignoraient pas qu'une grande partie de la zone torride était effectivement habitée. «Entre les tropiques, de nos jours, on est allé voir: on a constaté que la majeure partie en est habitable [...] Sur les 16800 stades ${ }^{81}$ qui séparent le tropique d'été de l'équateur, il y en a quelque 8800 qui ont été parcourus, et les informations sur ces régions ont été consignées à Alexandrie grâce aux souverains qui ont fait faire l'enquête [...] On voit par là que l'opinion qui veut que la région située entre les tropiques soit inhabitée par suite de l'excès de chaleur, et particulièrement la partie située au centre de la zone torride, est erreur notoire. " (Géminos, Intr. aux Phén. XVI, 24-25). De fait, la carte du monde habité proposée par Ératosthène s'étend au moins vers le $\mathrm{Sud}{ }^{82}$ jusqu'au parallèle situé à égale distance du tropique et de l'équateur $\left(12^{\circ} \mathrm{N}\right)$.

Bien plus, dans les milieux cultivés, l'opinion commençait à prévaloir que la zone équatoriale était plus tempérée que les zones tropicales. On s'était parfois demandé en effet si, dans la zone torride, la partie médiane n'était pas plus habitable

78 Héraclide du Pont (c.390-310), disciple de Platon, avait émis l'hypothèse que le Soleil était immobile et que la Terre circulait autour de lui. Toute son œuvre est perdue. Gélon (c. 540-478) devint tyran de Syracuse en 485; les mages étaient des savants babyloniens: ils seraient parvenus en Sicile après avoir fait le tour de l'Afrique et traversé le détroit de Gibraltar.

79 Eudoxe de Cyzique serait venu en Égypte comme ambassadeur sous le règne de Ptolémée Évergète II (regn. 146-117); il fit plusieurs tentatives dont Poseidonios ignorait le résultat final. Pline (II, 169) en dit le succès, sur la foi d'une déclaration de Cornelius Népos.

80 Cornelius Népos (c. 99-24 av. J.-C.), cité par Pline II, 169 et par Pomponius Mela III, 9, 90, soutenait que cet Eudoxe, parti du golfe Arabique, était parvenu jusqu'à Gadès en faisant le tour de l'Afrique.

81 Ces 16800 stades représentent 24 degrés de méridien. Même si personne n'avait jamais mesuré concrètement cette distance, l'évaluation en était facile depuis la mesure de la circonférence terrestre faite par Ératosthène: le degré de méridien y valait 700 stades; et pour la distance tropique-équateur, elle était fixée depuis longtemps à $1 / 15$ de grand cercle, même si Ératosthène avait trouvé une valeur meilleure. Mais les Grecs préféraient toujours user de chiffres ronds et d'approximations utiles.

82 Ptolémée, lui, étend le monde habité au sud de l'équateur, jusqu'au parallèle $16^{\circ} \mathrm{S}$, tandis que Marin de Tyr le prolongeait jusqu'au tropique d'hiver $\left(24^{\circ} \mathrm{S}\right)$. 
que les bords. Géminos rappelle à ce sujet que «Polybe l'historien ${ }^{83}$ a même composé un ouvrage intitulé Régions équatoriales, désignant par là les régions situées au centre de la zone torride. Il prétend que ces régions sont habitées et jouissent d'un climat plus tempéré que les contrées situées aux frontières de la zone torride. Il commence par faire état d'informations données par des témoins oculaires qui ont visité les lieux et expérimenté les apparences célestes; puis il raisonne en s'appuyant sur le mouvement naturel du soleil. Le soleil en effet reste longtemps aux entours des cercles tropiques ${ }^{84}$, dans son mouvement d'aller et retour, si bien que pendant quarante jours à peu près il séjourne sensiblement sur le cercle tropique; c'est d'ailleurs la raison pour laquelle la durée des jours reste sensiblement la même durant quarante jours. Le soleil s'attardant ainsi sur les lieux situés sous les tropiques, il est inévitable que de tels lieux soient calcinés et inhabitables du fait de l'excès de chaleur. À l'équateur en revanche, il se trouve que les déplacements sont rapides ${ }^{85}$; c'est pourquoi aussi la durée des jours subit de fortes variations au moment des équinoxes. Il est donc logique que les latitudes équatoriales soient plus tempérées, puisque le soleil, au lieu de s'attarder au zénith, s'en éloigne rapidement. Tous les lieux situés entre les tropiques sont bien dans une même position par rapport au trajet du soleil; seulement le soleil reste plus longtemps au zénith pour les latitudes proches des tropiques; c'est pourquoi les lieux situés sous l'équateur, en plein cœur de la zone torride, sont généralement plus tempérés que ceux situés aux frontières de la zone torride, c'est-à-dire sous les tropiques.» (Géminos, Intr. aux Phén. XVI, 32-38).

Avant Polybe, Ératosthène, après lui Poseidonios, avaient également soutenu l'opinion que l'équateur jouissait d'un climat plus tempéré que les zones tropicales. Poseidonios avait proposé de distinguer, «du point de vue des phénomènes humains, deux zones étroites situées sous les tropiques: le soleil y reste au zénith pendant à peu près la moitié d'un mois et elles sont divisées en deux par les tropiques. Ces zones ont ceci de particulier qu'elles sont complètement desséchées et recouvertes de sable, et qu'elles ne produisent que du silphium ${ }^{86}$ et quelques fruits aigres et recuits [...]

83 Polybe (c. 203-120), un Grec de Mégalopolis, est l'auteur d'une Histoire en trente-neuf livres qui retrace l'ascension irrésistible de la puissance romaine; séjournant à Rome comme otage après la défaite de la Ligue Achéenne, il noua des liens d'amitié avec la famille des Scipions, accompagna Scipion Émilien en Espagne et Numidie, revint avec lui en Afrique et explora les côtes de Mauritanie. Le livre XXXIV, perdu, était consacré à la géographie, avec des aperçus sur les continents et sur le peuplement de la zone équatoriale.

84 Les cercles tropiques en question sont les tropiques célestes. Pour les Grecs, les cercles fondamentaux de la sphère (équateur, tropiques, cercles arctiques) sont d'abord des cercles célestes, dont les cercles terrestres ne sont que la projection.

85 C'est aussi l'argument qu'avance Poseidonios, «que le déplacement du soleil sur le cercle oblique (sc. l'écliptique) y est plus rapide, de même que son mouvement d'orient en occident, car, parmi les mouvements accomplis à vitesse semblable, les plus rapides sont ceux qui se font sur le plus grand cercle» (Strabon, Géogr. II 3, 2).

86 Sur le silphium, une plante aujourd'hui disparue, qui avait fait la richesse de Cyrène, cf.F. C HAMOUX, Cyrène sous la monarchie des Battiades, Paris, 1952, p. 246-264. 
Manifestement, ajoute Poseidonios, ces caractères sont particuliers à ces zones, car, plus au sud, les conditions atmosphériques sont plus tempérées, et la terre plus fertile et mieux arrosée.» (Strabon, Géogr. II, 2, 3).Strabon critique cette manière d'introduire deux zones tropicales; il préfererait, lui, introduire une zone équatoriale. «Si, comme le dit Ératosthène, la région située sous l'équateur est tempérée (opinion partagée par Polybe [...]), mieux vaudrait définir une troisième zone tempérée, assez étroite, plutôt que d'introduire les zones tropicales.» (Géogr. II, 3, 2). Comme on peut voir, les discussions allaient bon train, dans l'Antiquité, à propos de la zone torride, de son extension et de son inaptitude prétendue à la vie humaine.

\section{Conclusion}

Les témoignages empruntés à des géographes des XVIe et XVII ${ }^{e}$ siècles montrent l'influence persistante de la culture classique, largement diffusée dans les écoles et les universités. D’où le désir de concilier les connaissances, théoriques plus que pratiques, des Anciens et les découvertes, théoriques mais surtout expérimentales, faites par les modernes. Tandis que Christophe Colomb faisait connaître de nouvelles terres, à l'Ouest, Nicolas Copernic (1473-1543) révolutionnait l'astronomie - jusqu'alors largement fondée sur l'hypothèse géocentrique - en mettant au point la théorie héliocentrique. Ce bouleversement des notions traditionnelles incitait les humanistes à chercher dans les textes anciens les signes avant-coureurs de ce que les modernes venaient de découvrir. Si l'on s'en tient au strict point de vue géographique ${ }^{87}$, on constate l'abondance des références aux auteurs latins ; si l'on citait quelques textes grecs ${ }^{88}$, plus ou moins authentiques, c'était le plus souvent à travers leur traduction latine. Et l'on sollicitait les œuvres littéraires plutôt que les traités véritablement scientifiques. Si Pline l'Ancien, avec son Histoire Naturelle, eut tant de succès au cours des siècles, c'est qu'il donnait à son lecteur l'illusion d'avoir consulté des sources nombreuses, grecques en particulier, et d'en reproduire plus ou moins fidèlement l'enseignement. La vague teinture scientifique ainsi obtenue dispensait de se plonger dans des lectures plus ardues.

À la question: si les Anciens connaissaient l'Amérique, on ne peut répondre, comme Mélétios d'Arta et d'autres, que par la négative. En revanche, ce que l'on peut affirmer sans crainte, c'est que, confiants dans ce que leur apprenait la géométrie de la sphère dans le cadre du géocentrisme, les Grecs étaient persuadés qu'il pouvait exister d'autres mondes habités que celui qu'ils connaissaient; ils n'excluaient nullement l'idée de les découvrir un jour, quand l'océan ne constituerait plus une barrière infranchissable. La découverte de l'aiguille aimantée au XIII ${ }^{\mathrm{e}}$ siècle permit de réaliser

87 Copernic aussi, dans le De Revolutionibus, invoquait des garants antiques.

88 Les savants grecs sont très peu sollicités. Ératosthène, Poseidonios, Géminos, Ptolémée ne sont jamais nommés (Strabon l'est peu), au profit des philosophes Platon, Aristote, Proclus. 
ce rêve, alimenté par l'histoire mythique de l'Atlantide. La circumnavigation de l'Afrique, les expéditions égyptiennes à la recherche des sources du Nil, la reconnaissance de l'île Taprobane-Ceylan, laissaient penser que la zone dite torride n'était pas inhabitable; Ptolémée d'ailleurs ne prolongeait-il pas jusqu'au sud de l'équateur un monde habité que l'on supposait jusqu'alors tout entier inclus dans l'hémisphère nord?

La mappemonde qui ouvrait la série des cartes dans la Géographie de Ptolémée, éditée à Ulm en 1482, montre l'Océan Indien encerclé de tous côtés par des terres. Quelque cent ans plus tard, la mappemonde d'Ortelius ${ }^{89}$, qui fait état de la découverte du Nouveau Monde, déploie sur une bonne partie de l'hémisphère sud, jusqu'au pôle, une "Terra australis nondum cognita», avec la Terre des Perroquets, sous le cap de Bonne Espérance, et la Terre de Feu, séparée de l'Amérique du Sud par le détroit de Magellan. On peut lire: "Ce continent austral, plus d'un le nomme pays de Magellan d'après son inventeur.» Ce fameux continent austral, dont l'existence avait été pressentie par les Anciens, fut ardemment recherché par les marins de toutes nationalités, jusqu'à ce que l'explorateur anglais James Cook (1728-1779), patrouillant en 1772-1775 dans le sud de l'Océan Indien et du Pacifique, finisse par assurer: "J'ai fait le tour de l'hémisphère austral dans une haute latitude et je l'ai traversé de manière à prouver, sans réplique, qu'il n'y a point de continent, à moins qu'il ne soit près du pôle et hors de portée des navigateurs.» Comme l'Atlantide, le continent austral avait vécu. Mais comme l'Atlantide et les continents mythiques imaginés par les Anciens, il avait donné l'espoir de découvrir des terres inconnues, et le courage de partir à leur recherche.

Germaine AUJAC

Université de Toulouse-Le Mirail

6, Boulevard Joffre

F-92340 Bourg-La-Reine

89 Cf. ORTELIUS, Théatre du Monde, Anvers, 1570. 